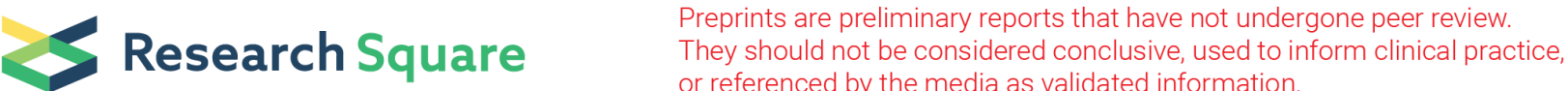

\section{ENPP1 is correlated with insulin resistance and lipid metabolism disorders in the rat model of polycystic ovary syndrome}

\author{
Jing Xia \\ Wuhan University \\ Yiqing Yang \\ Wuhan University \\ Zhe Yang \\ Wuhan University \\ Gengxiang Wu \\ Wuhan University \\ Jing Yang ( $\nabla$ dryangjing@whu.edu.cn ) \\ Wuhan University https://orcid.org/0000-0002-3286-0772
}

\section{Research Article}

Keywords: Polycystic ovary syndrome, Ectonucleotide pyrophosphatase phosphodiesterase 1, Insulin resistance, Lipid metabolism

Posted Date: December 9th, 2021

DOI: https://doi.org/10.21203/rs.3.rs-1136703/v1

License: (c) (i) This work is licensed under a Creative Commons Attribution 4.0 International License. Read Full License 


\section{Abstract \\ Purpose}

Previous studies have shown that ectonucleotide pyrophosphatase phosphodiesterase 1 (ENPP1) may be an inhibitor of the insulin signalling pathway, and insulin resistance (IR) is believed to be the core mechanism in the pathophysiology of polycystic ovary syndrome (PCOS). This study was aimed to investigate the expression of ENPP1 in different tissues of PCOS rats and to analyse its potential role in the pathophysiology of PCOS.

\section{Methods}

Eighteen 23-day-old Sprague-Dawley rats were divided into PCOS and control groups ( $n=9 /$ group). Samples were collected after 20 days. Pathological examination and immunofluorescence were performed. Western blotting results of ENPP1 in the ovaries were analysed. Serum levels of ENPP1, hormones, fasting blood glucose (FBG), serum lipids were measured.

\section{Results}

Levels of ENPP1, testosterone (T), FBG, fasting insulin (FINS), homeostasis model assessment of IR (HOMA-IR), free fatty acids (FFAs), leptin, cholesterol (TC), triglyceride (TG), and low-density lipoprotein cholesterol (LDL-C) were significantly higher in PCOS group, while adiponectin (ADP) and high-density lipoprotein cholesterol (HDL-C) were significantly lower. Spearman's rank correlation analysis showed that ENPP1 was correlated with T, HOMA-IR, FFAs, leptin, serum lipids and ADP. MRNA levels of ENPP1, BAX, and IRS1 were higher in the ovaries, skeletal muscle, subcutaneous fat, and visceral fat of PCOS rats, and the protein expression of ENPP1 was significantly higher in the ovaries.

\section{Conclusions}

Our results revealed that ENPP1 is highly associated with IR and lipid metabolism-related molecules, which may have play important roles in PCOS pathophysiological changes.

\section{Introduction}

Polycystic ovary syndrome (PCOS) is the most common endocrine condition in women of reproductive age, with a prevalence of 5 to $10 \%{ }^{[1]}$. Menstrual abnormalities, symptoms of androgen excess, and obesity are some of the clinical symptoms of PCOS. Apart from that, patients with PCOS also exhibit metabolic abnormalities, such as insulin resistance (IR), metabolic syndrome, hyperinsulinaemia, and dyslipidaemia ${ }^{[2,3]}$. According to previous reports, approximately $77 \%$ of patients with PCOS had IR, while 
$10 \%$ of those patients had type 2 diabetes $(T 2 D)^{[4,5]}$. In addition, the prevalence of metabolic syndrome in women with PCOS was increased by $43 \%^{[6]}$. Although the pathogenesis mechanism has not been well defined, IR is believed to be an important pathogenic factor ${ }^{[7]}$.

Ectonucleotide pyrophosphatase phosphodiesterase 1 (ENPP1), also known as plasma cell membrane glycoprotein 1 (PC-1), is a type II transmembrane glycoprotein containing pyrophosphatase and phosphodiesterase enzymatic activity and is highly expressed in bone and cartilage ${ }^{[8]}$. Scientists postulated a role for ENPP1 in the insulin signalling pathway for the first time in 1995, concluding that overexpression of ENPP1 dramatically reduces insulin receptor function ${ }^{[9]}$. ENPP1 binds to the insulin receptor $\beta$-subunit and affects IR signalling by blocking its autophosphorylation, thereby blocking the phosphorylation of insulin receptor substrate-1 and glucose transport, inhibiting the insulin signal transduction pathway, and contributing to the development of $\mathrm{IR}^{[10-12]}$. Apart from that, ENPP1 also modulates obesity, T2D, and diabetic consequences by affecting IR function in many tissues and cells, such as muscle and adipose tissue, fibroblasts and osteoblasts ${ }^{[13-15]}$. Therefore, in recent years, many scientists have investigated the role of ENPP1 in T2D and found its polymorphisms may be highly associated with T2D susceptibility and IR ${ }^{[16-18]}$.

Disturbance of lipid metabolism is one of the manifestations of PCOS ${ }^{[19]}$. Studies have shown that overexpression of ENPP1 in adipocytes induces fatty liver, hyperlipidaemia, and dysglycaemia ${ }^{[14]}$. In addition, genetic variants of the ENPP1 gene were associated with hypertriglyceridaemia in men and may contribute to the development of metabolic syndrome in this population ${ }^{[20]}$.

Considering ENPP1 may be an inhibitor of the insulin signalling pathway and associated with hyperlipidaemia, the present study was aimed at investigating the potential roles in the pathophysiology of PCOS via a dehydroepiandrosterone (DHEA)-induced rat PCOS model.

\section{Materials And Methods}

\section{Animals}

Eighteen female Sprague-Dawley adult rats (aged 21 days, weight 70-90 g) were purchased from the Laboratory Animal Centre of Wuhan University (No.430727201101344525, Wuhan, China). The rats were allowed to adapt to the environment for two days. All animals were grouped and housed in a room (22 2 ${ }^{\circ} \mathrm{C}, 12-\mathrm{h}$ light/12-h dark cycle) with free access to food and water.

Rats model was induced according to previous studies ${ }^{[21,22]}$. Briefly, rats were randomly divided into PCOS group ( $n=9)$ and control group $(n=9)$. The animals in the PCOS group were subcutaneously injected with 6 mg/100 g DHEA (Aladdin Reagent Co., Ltd., Shanghai, China), which was dissolved in olive oil, once a day for 20 consecutive days. The animals in the control group received subcutaneous injections of equivalent olive oil alone. 
The procedures involving rats and their care were carried out in accordance with the $\mathrm{NIH}$ guidelines (NIH Pub. No. 85-23, revised 1996). The experimenter obtained the certificate of qualification of laboratory animal professional technical examination (W20190028). The study was approved by the Laboratory Animal Welfare \& Ethics Committee of Renmin Hospital of Wuhan University (IACUC Issue No. WDRM 20200909).

\section{Sample collection}

After the last administration, all the rats fasted for $12 \mathrm{~h}$. Then, all the rats were anaesthetized with isoflurane ( $5 \%$ induction, $1 \%$ maintenance) in the early morning of the following day. Immediately after opening the abdominal cavity, for each rat, one ovary was removed and fixed in $4 \%$ paraformaldehyde, followed by paraffin embedding, and the other ovary was immediately stored at - 80 ${ }^{\circ} \mathrm{C}$. Then, the subcutaneous fat and visceral fat were separated. After cutting the leg skin, the skeletal muscles were separated. Two pieces of skeletal muscle, subcutaneous fat and visceral fat, each weighing approximately $2 \mathrm{~g}$, were collected from each animal. One was immediately fixed in $4 \%$ paraformaldehyde, followed by paraffin embedding, and the other was immediately stored at - 80 ${ }^{\circ} \mathrm{C}$. After tissue collection was completed, the chest was opened. Blood was extracted from the heart, placed on ice for 20 minutes, and then separated by centrifugation at $3000 \mathrm{rpm}$ for 20 minutes. The upper layer of serum was collected and stored at $-80^{\circ} \mathrm{C}$.

\section{Serum analysis}

According to the manufacturer's instructions, ENPP1 was detected by enzyme-linked immunosorbent assay (ELISA) kits (JYM1287Ra, Jiyinmei, Wuhan, China). Follicle-stimulating hormone (FSH) (RA20044), luteinizing hormone (LH) (RA20133), testosterone (T) (RA20653), fasting insulin (FINS) (RA20092), adiponectin (ADP) (RA20554), leptin (RA20489), and free fatty acids (FFA) (RA20313) concentrations of serum were detected by ELISA kits (Bioswamp, Wuhan, China). Fasting blood glucose (FBG) (F006-1-1), total cholesterol (TC) (A111-1-1), triglyceride (TG) (A110-1-1), high-density lipoprotein cholesterol (HDL-C) (A112-1-1), and low-density lipoprotein cholesterol (LDL-C) (A113-1-1) were detected by enzymatic colorimetric kits (Nanjing Jiancheng Bioengineering Institute, Nanjing, China). The homeostasis model assessment of IR (HOMA-IR) was calculated using the following formula: HOMA-IR= [FBG $(\mathrm{mmol} / \mathrm{l}) \times$ FINS (mU/l)]/22.5 ${ }^{[23] . ~}$

\section{Haematoxylin-eosin (H\&E) staining}

Ovaries, skeletal muscles, subcutaneous fat, and visceral fat were embedded in paraffin, serially sliced into 4- $\mu \mathrm{m}$-thick sections, and stained with H\&E according to the operating instructions (Servicebio, Beijing, 
China). The above sections were observed under a microscope (NanoZoomer S360, Hamamastu, Japan). ImageJ software (Version 1.53a; NIH, Bethesda, MD, USA) was used to analyse the cross-sectional area (CSA) of skeletal muscles and the mean cell area of subcutaneous fat and visceral fat.

\section{Quantitative reverse-transcription polymerase chain reaction (qRT-PCR)}

RNA was extracted from ovaries, skeletal muscle, subcutaneous fat, and visceral fat of rats by TRIzol (Accurate Biology, Changsha, China). Reverse transcription and qRT-PCR were performed using Evo M-MLV RT Mix Kit and qPCR Kit (Accurate Biology, Changsha, China). The specific primers were listed in Table 1. GAPDH served as an internal reference. The amplification conditions were as follows: an initial denaturation at $95^{\circ} \mathrm{C}$ for $30 \mathrm{~s}$, followed by 40 cycles of $95^{\circ} \mathrm{C}$ for $5 \mathrm{~s}$ and $60{ }^{\circ} \mathrm{C}$ for $30 \mathrm{~s}$.

\section{Immunofluorescence (IF) staining}

Sections of ovaries were used for IF staining following a previously described protocol ${ }^{[24]}$. Briefly, paraffin sections were incubated with $10 \%$ goat serum in PBS for 30 min to block nonspecific binding of the antibody. Then, the sections were incubated with 1:800 rabbit anti-rat ENPP1 antibody (Bioss, Beijing, China) overnight at $4{ }^{\circ} \mathrm{C}$. Fluorescein isothiocyanate (FITC) conjugated goat anti-rabbit lgG was used as secondary antibody for $2 \mathrm{~h}$ at room temperature. Nuclei were counterstained with 4',6-diamidino-2phenylindole (DAPI) (Servicebio, Beijing, China) at a dilution of 1:500 for 5 min. An Olympus laser scanning confocal microscope (BX53) was used for observation and photography.

\section{Western blotting}

Proteins of ovarian tissues were extracted in radio immunoprecipitation assay (RIPA) buffer in the presence of phosphatase and protease inhibitors. After separation by sulfate-polyacrylamide gel electrophoresis (SDS-PAGE) gels, protein samples were transferred onto polyvinylidene fluoride (PVDF) membranes. After $5 \%$ non-fat milk was added, the membranes were blocked at room temperature. The membranes were incubated with 1:2000 rabbit anti-rat ENPP1 antibody (Bioss, Beijing, China) overnight at $4{ }^{\circ} \mathrm{C}$. Subsequently, the membranes were incubated with horseradish peroxidase-conjugated secondary antibodies (Servicebio, Beijing, China) at room temperature for $1 \mathrm{~h}$. The protein bands were visualized by chemiluminescence reagent (Servicebio, Beijing, China) and analysed by ImageJ software.

\section{Statistical analysis}


All data were analysed using SPSS 26.0 statistical software (IBM, Armonk, NY, USA) and expressed as the mean \pm standard deviation (SD). Differences between the two groups were compared using unpaired ttests (data with normal distribution) or Mann-Whitney U tests (data with a skewed distribution). Spearman's rank correlation analysis was used to analyse the rank correlation between data. Software Graphpad Prism version 8.4 (GraphPad Software, San Diego, California) was used to plot bar graphs. Differences between groups with $\mathrm{P}<0.05$ were considered significant.

\section{Results}

\section{Pathological observations}

H\&E staining showed reduced granulosa cell layers, increased theca cell layers, more follicular cysts and atretic follicles, fewer follicles at all levels, and fewer corpora lutea in the ovaries of the PCOS group compared with the control group (Figure 1A). The mean CSA of skeletal muscle, the mean area of visceral fat cells and subcutaneous fat cells showed significantly greater in PCOS rats than control rats (Figure $1 B-G)$.

\section{Hormonal and biochemical indicators in serum}

The serum levels of FSH, LH, T, ENPP1, FINS, FBG and HOMA-IR of the PCOS group were significantly higher than control group (for FSH, $\mathrm{P}<0.001$; for $\mathrm{LH}, \mathrm{P}<0.001$; for $\mathrm{T}, \mathrm{P}<0.001$; for ENPP1, $\mathrm{P}<0.001$; for FINS, $\mathrm{P}<0.001$; for FBG, $\mathrm{P}=0.035$; for HOMA-IR, $\mathrm{P}<0.001$ ) (Figure 2). In addition, the PCOS group had significantly higher serum levels of FFA, leptin, TC, TG, LDL-C and significantly lower ADP and HDL-C levels than the control group (for FFA, $P=0.001$; for leptin, $P<0.001$; for $T C, P<0.001$; for $T G, P<0.001$; for $L D L-C, P<0.001$; for ADP, $P=0.002$; for HDL-C, $P<0.001$ ) (Figure 3).

\section{Correlation of serum ENPP1 with hormones and molecules}

The correlation of ENPP1 with HOMA-IR, hormones and lipid levels were studied (Table 2,3). The results of Spearman's rank correlation analysis showed that ENPP1 had strong positive correlations with T, HOMA-IR, FFA, leptin, TC, TG, and LDL-C (for T, $P<0.001$; for HOMA-IR, $P=0.012$; for FFA, $P=0.018$; for leptin, $\mathrm{P}=0.002$; for $\mathrm{TC}, \mathrm{P}<0.001$; for $\mathrm{TG}, \mathrm{P}=0.002$; for $\mathrm{LDL}-\mathrm{C}, \mathrm{P}<0.001$ ). In addition, ENPP1 also showed a strong negative correlation with ADP $(P=0.003)$. However, there was no significant correlation between ENPP1 and HDL-C.

\section{Expression of mRNAs in ovaries, skeletal muscles, visceral fat, and subcutaneous fat}


In ovaries and skeletal muscles, ENPP1 mRNA, BAX mRNA, and IRS1 mRNA were significantly higher in PCOS group than those in control group $(P<0.05)$ (Figure 4A, B). In visceral fat, ENPP1 mRNA was significantly higher in the PCOS group $(P<0.05)$. Although BAX mRNA and IRS1 mRNA levels were higher in the PCOS group, no significant difference was found (Figure $4 C$ ). In addition, in subcutaneous fat, ENPP1 mRNA and BAX mRNA were significantly higher in the PCOS group than those in the control group $(P<0.05)$. Although IRS1 mRNA was higher in the PCOS group, the difference was not significant (Figure 4D).

\section{Expression of ENPP1 in ovarian tissues}

IF staining of ovaries showed that ENPP1 was mainly expressed in the cytoplasm and cell membrane (Figure 4E). In addition, western blotting analysis showed that the expression level of ENPP1 was significantly higher in the ovaries of the PCOS group than in the control group $(P<0.05)$ (Figure 4F, $G$ ).

\section{Discussion}

In this study, DHEA-treated rats were used to simulate endocrine disorders and ovarian pathological changes in PCOS. The ovaries of PCOS rats showed polycystic changes. The CSA of skeletal muscle and the mean cell area of visceral fat and subcutaneous fat were significantly greater in PCOS rats than in control rats. We found that ENPP1 was expressed at higher levels in different tissues in PCOS rats than in control rats, such as ovaries, skeletal muscles, visceral fat, and subcutaneous fat. The serum levels of ENPP1, FBG, FINS, FFA, leptin, TC, TG, and LDL-C were significantly higher, and the levels of ADP and HDL-C were significantly lower in PCOS group than in the control group. In addition, the serum concentration of ENPP1 was highly correlated with levels of T, HOMA-IR and serum lipids.

Androgens are well known to increase muscle strength and mass, and muscle size is positively correlated with serum androgen levels in women with PCOS ${ }^{[25]}$. The DHEA rats in this study showed increased serum T levels which mimicked the increased androgens in PCOS patients, and it led to an increase in skeletal muscle fibre CSA, which represents muscle hypertrophy. In addition, excess androgens can also lead to visceral obesity, i.e., hypertrophy of visceral and subcutaneous fat cells, as verified in this study, as well as dyslipidaemia. It is believed that androgen excess leads to PCOS exhibiting IR and visceral obesity and further promotes androgen secretion from the ovaries and adrenal glands, thus fostering a vicious cycle of PCOS ${ }^{[26]}$.

The abnormal expression of indicators in the serum of PCOS rats reflected the possible existence of IR and lipid metabolism disorders in PCOS. FFAs have a close relationship with IR because FFAs are involved in the production of lipid metabolites, proinflammatory cytokines (TNF- $a$, IL-1 $1 \beta$, IL-6, MCP-1), and cellular stress, such as oxidative and endoplasmic reticulum stress ${ }^{[27]}$. The above processes may cause the development of IR. In addition, ADP and leptin were reported to be associated with IR, independent of obesity ${ }^{[28]}$. Moreover, it was reported that PCOS patients had considerably higher TG/HDL-C, TC/HDL-C, 
and LDL-C/HDL-C ratios than age-matched healthy women and a strong positive connection with HOMA$\mathrm{IR}^{[29]}$. The same results were found in our study. In addition, Spearman's rank correlation analysis showed a strong correlation between ENPP1 and almost all of the detected indicators, which suggested that ENPP1 was highly correlated with IR and lipid metabolism disorders in PCOS.

Some scientists have proposed a possible mechanism of action of ENPP1 in IR. A study suggested that when the missense variant K121 of the ENPP1 gene occurred, in which lysine (K) was replaced by glutamine $(\mathrm{Q})$, it predisposed patients to $\mathrm{IR}^{[30]}$. ENPP1 carrying the Q121 variant had increased physical interaction with the insulin receptor at the cell membrane and became a stronger inhibitor of insulin signalling than ENPP1 carrying K121. This further affected insulin-stimulated receptor autophosphorylation and subsequent stimulation of IRS1 phosphorylation, PI3-kinase activation, and glycogen synthesis. It has been shown that the K121Q variant in the ENPP1 gene is significantly linked to PCOS risk ${ }^{[31]}$. Our research will continue to explore the relationship between the expression level of ENPP1 and PCOS.

ENPP1 is significantly upregulated in cancerous tissues, may be an initiator of increases cell proliferation, migration, and invasion ${ }^{[8,32]}$. Compared with the control group, we found that ENPP1 levels were significantly higher in both ovaries and serum of PCOS rats, and ENPP1 was mainly expressed in the cytoplasm and cell membrane in ovaries, especially highly expressed in the granulosa cells of PCOS rats, suggesting that elevated ENPP1 was associated with higher proliferation rates in ovarian granulosa cells of PCOS rats.

IRS1 is a major protein involved in insulin signalling that activates several signalling pathways involved in the regulation of glucose uptake, protein synthesis and gene expression ${ }^{[33]}$. BAX is a key proapoptotic protein of the BCL-2 family and a major regulator of the mitochondrial pathway ${ }^{[34]}$. In ovary, skeletal muscle, subcutaneous fat and visceral fat, ENPP1 mRNA levels were higher in PCOS rats than in controls and significantly correlated with the HOMA-IR index. In addition, mRNA levels of BAX and IRS1 were also significantly higher in ovary and skeletal muscle in PCOS rats, and ENPP1 levels were also significantly correlated with them. These findings suggested that ENPP1 was highly associated with insulin pathway (showed by results of IRS1) in the ovary and the apoptosis (showed by results of BAX) of ovarian granulosa cells. Meanwhile, it may also be involved in the process of IR in skeletal muscle, subcutaneous fat and visceral fat, thus changing the general state of PCOS rats.

One of the limitations of our study is that this is only a preliminarily study about the correlation between ENPP1, IR and lipid metabolism-related molecules. The mechanism of ENPP1 in PCOS has not yet been determined. Second, the rats modelled by DHEA cannot fully simulate all the physiological characteristics of PCOS patients. Therefore, our next research plan is to analyse the expression of ENPP1 in human specimens and regulate the expression of ENPP1 in granulosa cells to further explore the role of ENPP1 in pathology and pathological processes in PCOS.

\section{Conclusion}


ENPP1 expression was significantly higher in the ovaries, skeletal muscle, subcutaneous fat, and serum of PCOS rats. The abnormal expression of ENPP1 was highly correlated with insulin resistance and lipid metabolism-related molecules, suggesting that ENPP1 may play an important role in the process of insulin resistance in PCOS.

\section{Declarations}

\section{ACKNOWLEDGMENTS:}

\section{Funding}

This work was financed by the National Natural Science Foundation of China (grant numbers 81873817, $81883718,81971356)$. The authors declare no conflict of interest.

\section{Author Contributions}

GXW and JY led the entire project. JX evaluated all data. JX, YQY and ZY designed and performed the experiments. JX wrote the manuscript. All authors have read and approved the final version of the manuscript.

\section{Disclosure}

The authors have no competing interests to declare.

\section{References}

1. Ożegowska K, Plewa S, Mantaj U, Pawelczyk L, Matysiak J. Serum Metabolomics in PCOS Women with Different Body Mass Index. J. Clin. Med. 2021;10(13): 2811. doi: 10.3390/jcm10132811.

2. Rotterdam ESHRE/ASRM-Sponsored PCOS Consensus Workshop Group. Revised 2003 consensus on diagnostic criteria and long-term health risks related to polycystic ovary syndrome. Fertil. Steril. 2004;81(1):19-25. doi: 10.1016/j.fertnstert.2003.10.004.

3. Di Pietro M, Parborell F, Irusta G, Pascuali N, Bas D, Bianchi MS, Tesone M, Abramovich D. Metformin regulates ovarian angiogenesis and follicular development in a female polycystic ovary syndrome rat model. Endocrinology. 2015;156(4):1453-1463. doi: 10.1210/en.2014-1765.

4. Tao T, Wu P, Wang Y, Liu W. Comparison of glycemic control and $\beta$-cell function in new onset T2DM patients with PCOS of metformin and saxagliptin monotherapy or combination treatment. BMC Endocr. Disord. 2018;18(1):14. doi: 10.1186/s12902-018-0243-5. 
5. Li W, Chen Q, Xie Y, Hu J, Yang S, Lin M. Prevalence and degree of insulin resistance in Chinese Han women with PCOS: Results from euglycemic-hyperinsulinemic clamps. Clin. Endocrinol. (Oxf). 2019;90(1):138-144. doi: 10.1111/cen.13860.

6. Zhang Y, Li C, Zhang W, Zheng X, Chen X. Decreased Insulin Resistance by Myo-Inositol Is Associated with Suppressed Interleukin 6/Phospho-STAT3 Signaling in a Rat Polycystic Ovary Syndrome Model. J. Med. Food. 2020;23(4):375-387. doi: 10.1089/jmf.2019.4580.

7. Calcaterra V, Verduci E, Cena H, Magenes VC, Todisco CF, Tenuta E, Gregorio C, De Giuseppe R, Bosetti A, Di Profio E, Zuccotti G. Polycystic Ovary Syndrome in Insulin-Resistant Adolescents with Obesity: The Role of Nutrition Therapy and Food Supplements as a Strategy to Protect Fertility. Nutrients. 2021;13(6): 1848. doi: 10.3390/nu13061848.

8. Hu M, Guo W, Liao Y, Xu D, Sun B, Song H, Wang T, Kuang Y, Jing B, Li K, Ling J, Yao F, Deng J. Dysregulated ENPP1 increases the malignancy of human lung cancer by inducing epithelialmesenchymal transition phenotypes and stem cell features. Am. J. Cancer Res. 2019;9(1):134-144.

9. Maddux BA, Sbraccia P, Kumakura S, Sasson S, Youngren J, Fisher A, Spencer S, Grupe A, Henzel W, Stewart TA. Membrane glycoprotein PC-1 and insulin resistance in non-insulin-dependent diabetes mellitus. Nature. 1995;373(6513):448-451. doi: 10.1038/373448a0.

10. Tassone EJ, Cimellaro A, Perticone M, Hribal ML, Sciacqua A, Andreozzi F, Sesti G, Perticone F. Uric Acid Impairs Insulin Signaling by Promoting Enpp1 Binding to Insulin Receptor in Human Umbilical Vein Endothelial Cells. Front. Endocrinol. (Lausanne). 2018;9:98. doi: 10.3389/fendo.2018.00098.

11. Marucci A, Antonucci A, De Bonis C, Mangiacotti D, Scarale MG, Trischitta V, Di Paola R. GALNT2 as a novel modulator of adipogenesis and adipocyte insulin signaling. Int. J. Obes. (Lond). 2019;43(12):2448-2457. doi: 10.1038/s41366-019-0367-3.

12. Tumurbaatar B, Poole AT, Olson G, Makhlouf M, Sallam HS, Thukuntla S, Kankanala S, Ekhaese O, Gomez G, Chandalia M, Abate N. Adipose Tissue Insulin Resistance in Gestational Diabetes. Metab. Syndr. Relat. Disord. 2017;15(2):86-92. doi: 10.1089/met.2016.0124.

13. Goldfine ID, Maddux BA, Youngren JF, Reaven G, Accili D, Trischitta V, Vigneri R, Frittitta L. The role of membrane glycoprotein plasma cell antigen 1/ectonucleotide pyrophosphatase phosphodiesterase 1 in the pathogenesis of insulin resistance and related abnormalities. Endocr. Rev. 2008;29(1):62-75. doi: 10.1210/er.2007-0004.

14. Pan W, Ciociola E, Saraf M, Tumurbaatar B, Tuvdendorj D, Prasad S, Chandalia M, Abate N. Metabolic consequences of ENPP1 overexpression in adipose tissue. Am. J. Physiol. Endocrinol. Metab. 2011;301(5):E901-E911. doi: 10.1152/ajpendo.00087.2011. 
15. Huesa C, Zhu D, Glover JD, Ferron M, Karsenty G, Milne EM, Millan JL, Ahmed SF, Farquharson C, Morton NM, MacRae VE. Deficiency of the bone mineralization inhibitor NPP1 protects mice against obesity and diabetes. Dis. Model. Mech. 2014;7(12):1341-1350. doi: 10.1242/dmm.017905.

16. Chen L, Qin Y, Liang D, Liang X, Liang Y, Li L, Xian J, Zhang L, Tong L, Li H, Zhang H. Gender differences in the association of ENPP1 polymorphisms with type 2 diabetes in a Chinese population. Gene. 2017;637:190-195. doi: 10.1016/j.gene.2017.09.052.

17. Hsiao T-J, Lin E. The ENPP1 K121Q polymorphism is associated with type 2 diabetes and related metabolic phenotypes in a Taiwanese population. Mol. Cell. Endocrinol. 2016;433:20-25. doi: 10.1016/j.mce.2016.05.020.

18. Marchenko IV, Dubovyk YI, Tkach GF, Maksymova OS, Matlai OI, Ataman AV, Harbuzova VY. The association between enpp1 rs997509 polymorphism and type 2 diabetes mellitus development in ukrainian population. Wiad Lek. 2018;71(3 pt 1):490-495.

19. Zhang J, Xu J-H, Qu Q-Q, Zhong G-Q. Risk of Cardiovascular and Cerebrovascular Events in Polycystic Ovarian Syndrome Women: A Meta-Analysis of Cohort Studies. Front. Cardiovasc. Med. 2020;7:552421. doi: 10.3389/fcvm.2020.552421.

20. Tanyolaç S, Bremer AA, Hodoglugil U, Movsesyan I, Pullinger CR, Heiner SW, Malloy MJ, Kane JP, Goldfine ID. Genetic variants of the ENPP1/PC-1 gene are associated with hypertriglyceridemia in male subjects. Metab. Syndr. Relat. Disord. 2009;7(6):543-548. doi: 10.1089/met.2009.0027.

21. Furat Rencber S, Kurnaz Ozbek S, Eraldemır C, Sezer Z, Kum T, Ceylan S, Guzel E. Effect of resveratrol and metformin on ovarian reserve and ultrastructure in PCOS: an experimental study. $J$. Ovarian Res. 2018;11(1):55. doi: 10.1186/s13048-018-0427-7.

22. Wu G, Hu X, Ding J, Yang J. Abnormal expression of HSP70 may contribute to PCOS pathology. J. Ovarian Res. 2019;12(1):74. doi: 10.1186/s13048-019-0548-7.

23. Huang X, Wang Q, Liu T, Pei T, Liu D, Zhu H, Huang W. Body fat indices as effective predictors of insulin resistance in obese/non-obese polycystic ovary syndrome women in the Southwest of China. Endocrine. 2019;65(1):81-85. doi: 10.1007/s12020-019-01912-1.

24. Khan MS, Muhammad T, Ikram M, Kim MO. Dietary Supplementation of the Antioxidant Curcumin Halts Systemic LPS-Induced Neuroinflammation-Associated Neurodegeneration and Memory/Synaptic Impairment via the JNK/NF-B/Akt Signaling Pathway in Adult Rats. Oxid. Med. Cell. Longev. 2019;2019:7860650. doi: 10.1155/2019/7860650.

25. DeChick A, Hetz R, Lee J, Speelman DL. Increased Skeletal Muscle Fiber Cross-Sectional Area, Muscle Phenotype Shift, and Altered Insulin Signaling in Rat Hindlimb Muscles in a Prenatally 
Androgenized Rat Model for Polycystic Ovary Syndrome. Int. J. Mol. Sci. 2020;21(21): 7918. doi: 10.3390/ijms21217918.

26. Guo Z, Chen X, Feng P, Yu Q. Short-term rapamycin administration elevated testosterone levels and exacerbated reproductive disorder in dehydroepiandrosterone-induced polycystic ovary syndrome mice. J. Ovarian Res. 2021;14(1):64. doi: 10.1186/s13048-021-00813-0.

27. Boden G. Obesity, insulin resistance and free fatty acids. Curr. Opin. Endocrinol. Diabetes Obes. 2011;18(2):139-143. doi: 10.1097/MED.0b013e3283444b09.

28. Polak K, Czyzyk A, Simoncini T, Meczekalski B. New markers of insulin resistance in polycystic ovary syndrome. J. Endocrinol. Invest. 2017;40(1):1-8. doi: 10.1007/s40618-016-0523-8.

29. Xiang S-K, Hua F, Tang Y, Jiang X-H, Zhuang Q, Qian F-J. Relationship between Serum Lipoprotein Ratios and Insulin Resistance in Polycystic Ovary Syndrome. Int. J. Endocrinol. 2012;2012:173281. doi: 10.1155/2012/173281.

30. Abate N, Chandalia M, Di Paola R, Foster DW, Grundy SM, Trischitta V. Mechanisms of disease: Ectonucleotide pyrophosphatase phosphodiesterase 1 as a 'gatekeeper' of insulin receptors. Nat. Clin. Pract. Endocrinol. Metab. 2006;2(12):694-701. doi: 10.1038/ncpendmet0367.

31. Heinonen S, Korhonen S, Helisalmi S, Koivunen R, Tapanainen JS, Laakso M. The 121Q allele of the plasma cell membrane glycoprotein 1 gene predisposes to polycystic ovary syndrome. Fertil. Steril. 2004;82(3):743-745. doi: 10.1016/j.fertnstert.2004.03.031.

32. Wang $\mathrm{H}, \mathrm{Ye} \mathrm{F}$, Zhou $\mathrm{C}$, Cheng Q, Chen H. High expression of ENPP1 in high-grade serous ovarian carcinoma predicts poor prognosis and as a molecular therapy target. PLOS One. 2021;16(2):e0245733. doi: 10.1371/journal.pone.0245733.

33. Zhuang J, Song Y, Ye Y, He S, Ma X, Zhang M, Ni J, Wang J, Xia W. PYCR1 interference inhibits cell growth and survival via c-Jun N-terminal kinase/insulin receptor substrate 1 (JNK/IRS1) pathway in hepatocellular cancer. J. Transl. Med. 2019;17(1):343. doi: 10.1186/s12967-019-2091-0.

34. Cosentino K, García-Sáez AJ. Bax and Bak Pores: Are We Closing the Circle? Trends Cell Biol. 2017;27(4):266-275. doi: 10.1016/j.tcb.2016.11.004.

\section{Tables}

Table 1 Primers of the genes used in qRT-PCR 


\begin{tabular}{ll}
\hline GAPDH-F & CGCTAACATCAAATGGGGTG \\
GAPDH-R & TTGCTGACAATCTTGAGGGAG \\
ENPP1-F & GAAAGACCACACTTTTACACTC \\
ENPP1-R & TTACAACTGCCTTGTTCCATGCC \\
BAX-F & GATGGCCTCCTTTCCTACTTC \\
BAX-R & CTTCTTCCAGATGGTGAGTGAG \\
IRS1-F & GCCTGGAGTATTATGAGAACGAGAA \\
IRS1-R & GGGGATCGAGCGTTTGG \\
\hline
\end{tabular}

Table 2 Spearman rank's analysis for the correlation of ENPP1 with HOMAIR and hormones

\begin{tabular}{|c|c|c|c|c|c|c|}
\hline \multirow{2}{*}{$\overline{\mathrm{T}}$} & \multirow[b]{2}{*}{$r$} & \multirow{2}{*}{$\frac{\text { ENPP1 }}{.785^{* *}}$} & \multirow[t]{2}{*}{$\mathrm{T}$} & \multicolumn{2}{|c|}{ HOMA-IRFFA } & \multirow[t]{2}{*}{$\mathrm{ADP}$} \\
\hline & & & & & & \\
\hline $\mathrm{P}$ & .00 & & & & & \\
\hline \multirow[t]{2}{*}{ HOMA-I } & & $.577 *$ & $.686^{* *}$ & & & \\
\hline & $\mathrm{P}$ & .012 & .002 & & & \\
\hline \multirow[t]{2}{*}{ FFA } & $\mathrm{r}$ & $.550 *$ & $.474 *$ & $.515^{*}$ & & \\
\hline & $\mathrm{P}$ & .018 & .047 & .029 & & \\
\hline \multirow[t]{2}{*}{$\mathrm{ADP}$} & $\mathrm{r}$ & $-.655^{* *}$ & $-.482 *$ & -.424 & -.453 & \\
\hline & $\mathrm{P}$ & .003 & .043 & .079 & .059 & \\
\hline \multirow[t]{2}{*}{ Leptin } & $\mathrm{r}$ & $.688^{* *}$ & $.738^{* *}$ & $.616^{* *}$ & $.839 * *$ & $.686 * *$ \\
\hline & $\mathrm{P}$ & .002 & .000 & .006 & .000 & .002 \\
\hline
\end{tabular}

$* \mathrm{P}<0.05, * * \mathrm{P}<0.01$ 
Table 3 Spearman rank's analysis for the correlation of ENPP1 with lipid levels.

\begin{tabular}{|c|c|c|c|}
\hline & ENPP1 TC & TG $\mathrm{H}$ & HDL-C \\
\hline \multirow[t]{2}{*}{ TC } & r . $779 * *$ & & \\
\hline & P .000 & & \\
\hline \multirow[t]{2}{*}{ TG } & r . $.680^{* *} .702^{* *}$ & & \\
\hline & $\begin{array}{lll}\text { P } & .002 & .001\end{array}$ & & \\
\hline \multirow[t]{2}{*}{ HDL- } & Cr $-. .451 \quad-.497 *$ & $.701 * *$ & \\
\hline & $\begin{array}{lll}P & .060 \quad .036\end{array}$ & .001 & \\
\hline \multirow[t]{2}{*}{ LDL- } & r $.755^{* *} .901 * *$ & $.664^{* *} .7$ & $.767 * *$ \\
\hline & $\begin{array}{lll}P & .000 \quad .000\end{array}$ & 3.000 & .000 \\
\hline
\end{tabular}

$* \mathrm{P}<0.05, * * \mathrm{P}<0.01$.

\section{Figures}

\section{Figure 1}

Pathological examination (H\&E staining). (A) Comparison of rat ovarian structure ( $\times 4$ magnification). PCOS group showed an increase in cystic follicles. Follicles and corpus luteum at different developmental stages were rare. The number of granulosa cells decreased to 2 to 3 layers, the follicular cell layer became thicker, and the follicular membrane cells proliferated. (B) skeletal muscle crosssections images ( $\times 200$ magnification); (C) Mean CSA of skeletal muscles; (D) visceral fat images $(\times 400$ magnification); (E) Mean area of visceral fat cells; (F) Subcutaneous fat images ( $\times 400$ magnification); (G) Mean area of subcutaneous fat cells. Data represent the mean \pm SD of three independent experiments. **: $P<0.001$. 
A

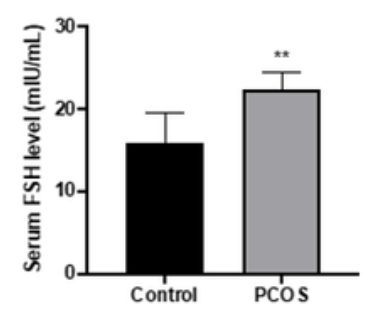

$\mathbf{E}$
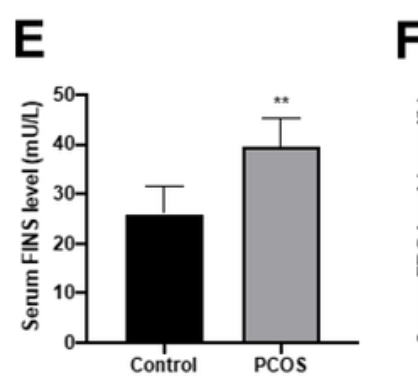

B

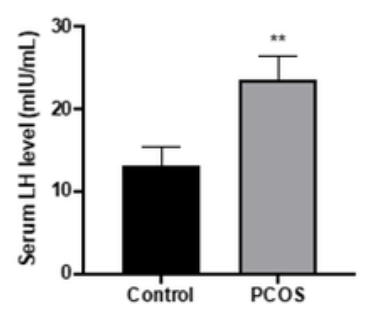

$\mathbf{F}$

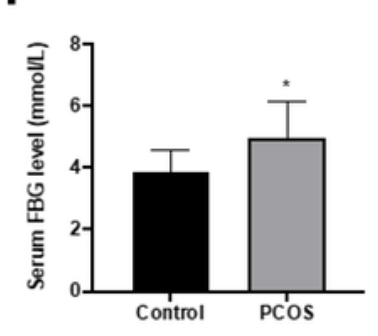

C

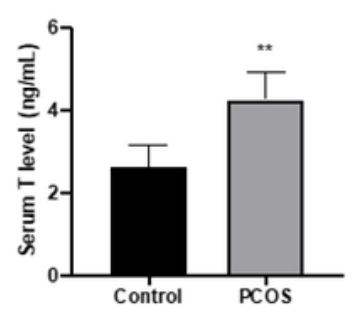

G

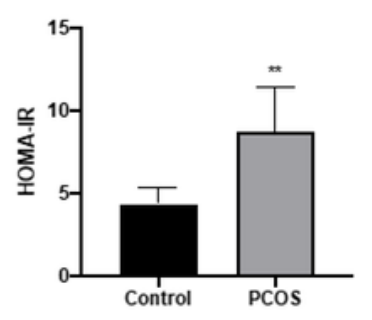

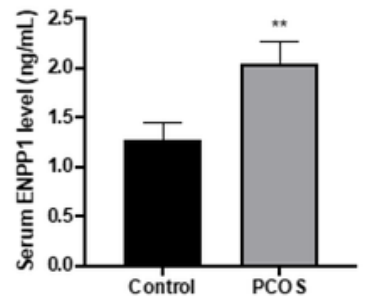

Figure 2

\section{Figure 2}

Concentrations of hormones and glucose metabolism indicators (mean $\pm S D, n=9$ per group). (A-E) Serum concentrations of FSH, LH, T, ENPP1, and FINS were determined by ELISA. (F) Levels of serum FBG were determined by enzymatic colorimetric kits. (G) HOMA-IR index of two groups. *: $\mathrm{P}<0.05$; $* *$ : $\mathrm{P}<0.001$. 
A

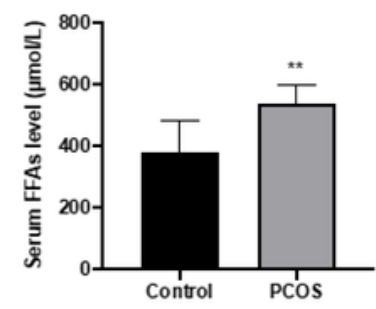

E

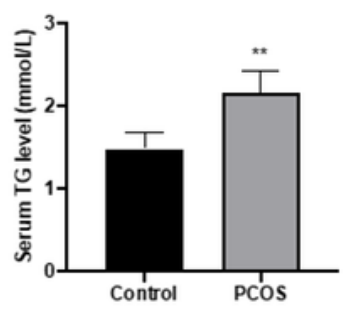

B

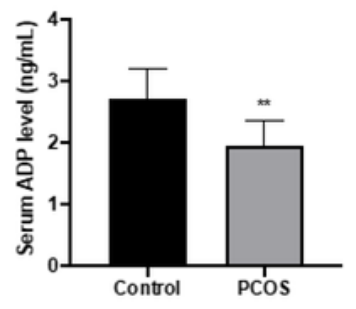

C

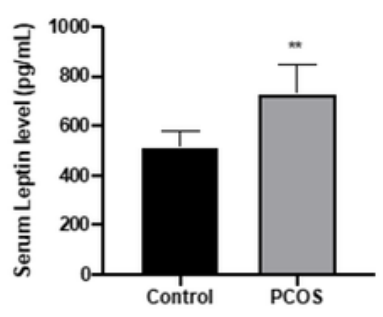

G
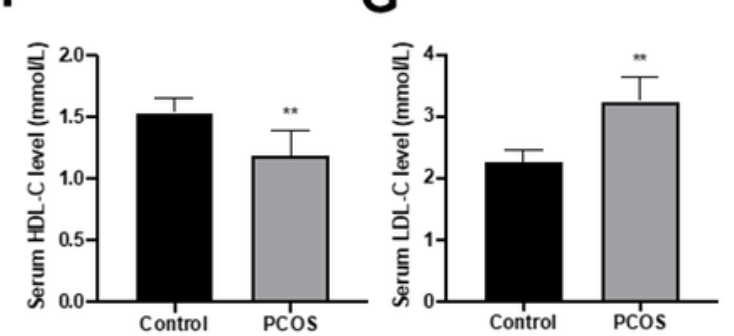

D

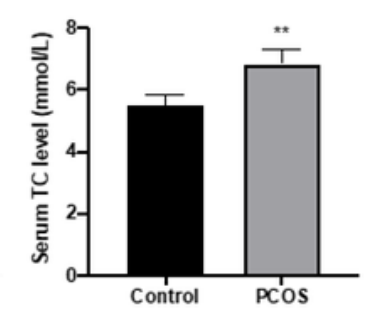

Figure 3

\section{Figure 3}

Serum levels of lipid-metabolism related molecules (mean $\pm S D, n=9$ per group). (A-C) Serum concentrations of FFA, ADP, and leptin were determined by ELISA. (D-G) Levels of serum TC, TG, HDL-C, LDL-C were determined by enzymatic colorimetric kits. **: $P<0.001$. 
A

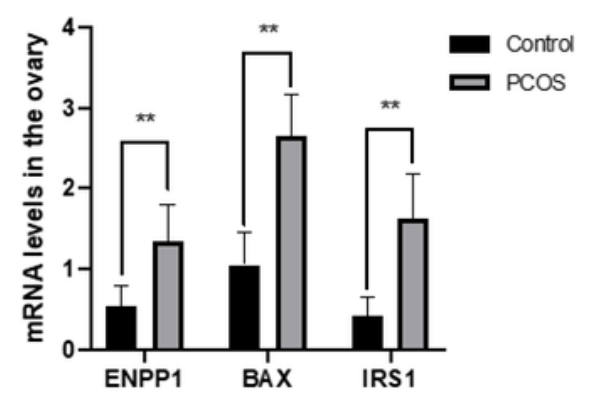

C

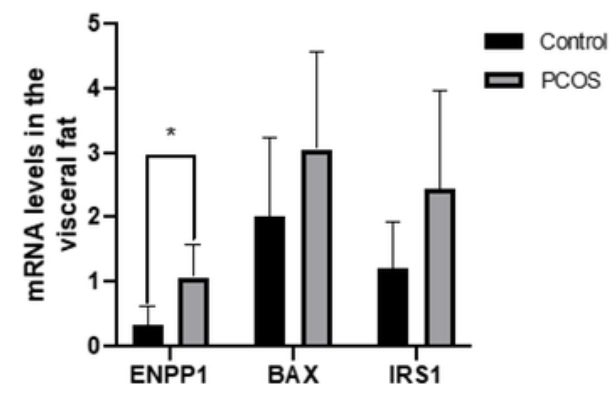

B

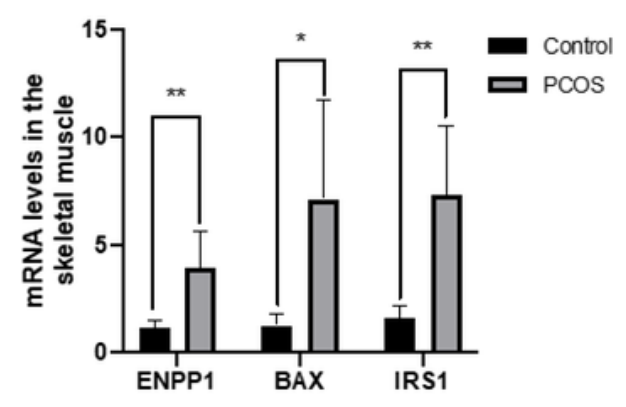

D

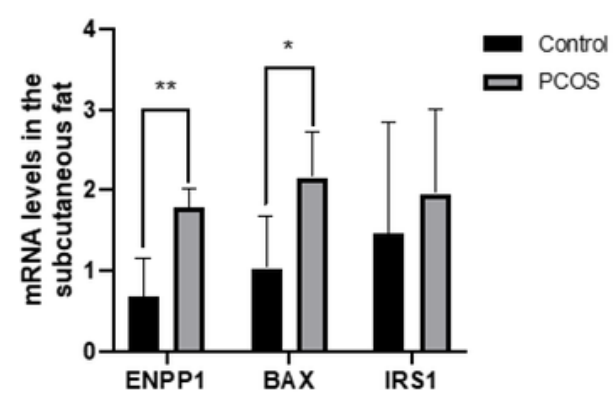

$\mathbf{E}$
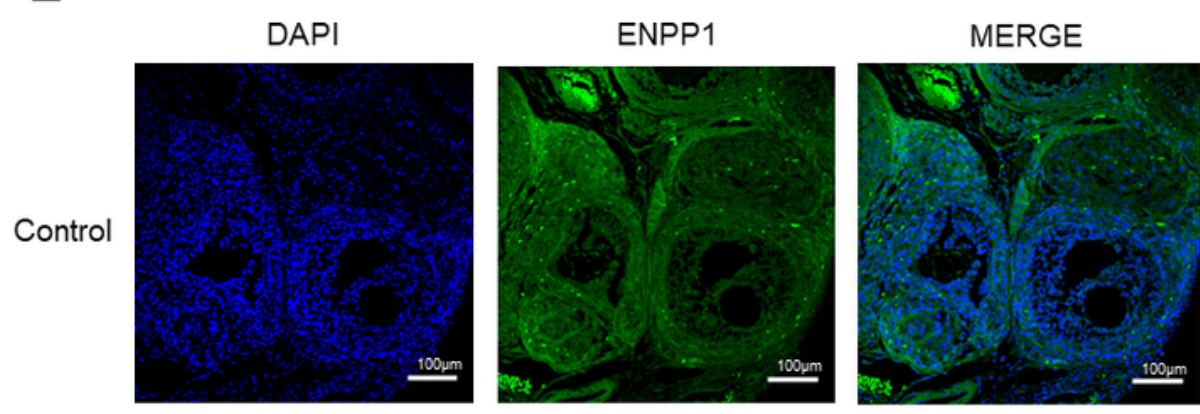

$\mathbf{F}$
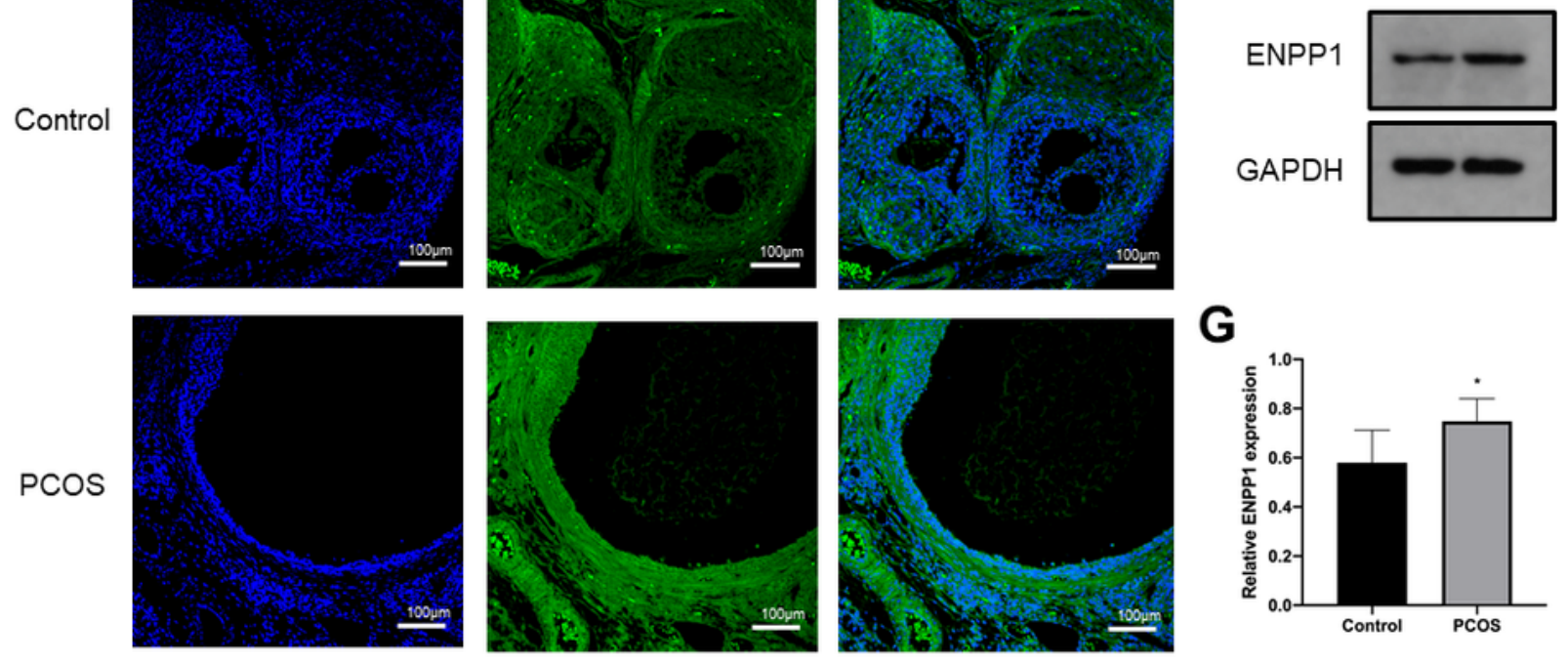

G

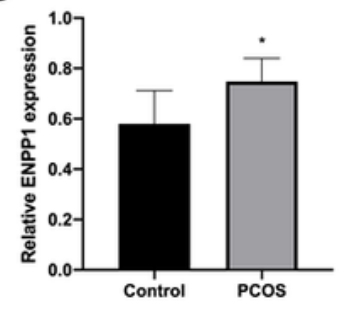

Figure 4

\section{Figure 4}

Expression of ENPP1 in tissues. (A-D) MRNA expression levels of ENPP1, BAX, IRS1 in ovarian tissues, skeletal muscles, visceral fat, and subcutaneous fat (mean $\pm S D, n=5$ per group). MRNA expression levels of ENPP1 of PCOS group was significantly higher than control group in these four tissues. MRNA levels of BAX and IRS1 in ovarian tissues and skeletal muscles, and mRNA levels of BAX in subcutaneous fat were significantly higher in PCOS group than control group. (E) The IF images of ENPP1 expression in 
ovarian tissues ( $\times 200$ magnification). Green color (FITC) and blue color (DAPI) represents the results of ENPP1 in the ovarian tissues. ENPP1 was mainly expressed in the cytoplasm and cell membrane. (F) Representative western blotting bands. (G) Bar graph represents the level of ENPP1, normalized to the total amount of GAPDH protein (mean $\pm S D, n=6$ per group). *: $P<0.05 ; * \star: P<0.001$. 